\title{
How to define quantum mean-field solvable Hamiltonians using Lie algebras
}

\author{
Artur F. Izmaylov ${ }^{1,2, *}$ and Tzu-Ching Yen $^{2}$ \\ ${ }^{1}$ Department of Physical and Environmental Sciences, \\ University of Toronto Scarborough, Toronto, Ontario, M1C 1A4, Canada \\ ${ }^{2}$ Chemical Physics Theory Group, Department of Chemistry, \\ University of Toronto, Toronto, Ontario, M5S 3H6, Canada
}

\begin{abstract}
Necessary and sufficient conditions for quantum Hamiltonians to be exactly solvable within meanfield theories have not been formulated so far. To resolve this problem, first, we define what meanfield theory is, independently of a Hamiltonian realization in a particular set of operators. Second, using a Lie-algebraic framework we formulate a criterion for a Hamiltonian to be mean-field solvable. The criterion is applicable for both distinguishable and indistinguishable particle cases. For the electronic Hamiltonians, our approach reveals the existence of mean-field solvable Hamiltonians of higher fermionic operator powers than quadratic. Some of the mean-field solvable Hamiltonians require different sets of quasi-particle rotations for different eigenstates, which reflects a more complicated structure of such Hamiltonians.
\end{abstract}

\section{INTRODUCTION}

Mean-field (MF) theories are useful for solving manybody problems in both classical and quantum computing. In classical computing, they provide an approximate description that can serve as a basis for further more accurate perturbational treatment. ${ }^{1}$ Another useful feature of MF procedures is the relative simplicity of their eigenstates, which can be thought of as the most general un-entangled states, ${ }^{2}$ and thus are easy to represent on a classical computer. In quantum computing, formulation of MF-solvable Hamiltonians allows one to use such Hamiltonians as elementary blocks in decomposing realistic Hamiltonians for efficient quantum measurements. ${ }^{3}$ Also, it was shown that MF transformations are computationally simple and can be efficiently implemented on both quantum and classical computers. ${ }^{4,5}$

MF theories can be formulated using different sets of operators to express many-body Hamiltonians, for example, fermionic creation and annihilation or qubit Pauli operators, with fermion-qubit transformations to switch between these operator sets. ${ }^{6,7}$ One may wonder what the common features of all MF treatments are, independent of the Hamiltonian operator expression? Usually, this question is addressed by introducing a notion of single particles and single-particle operators. Then an $N$ particle state is considered to be an MF-state if it is an eigenstate of a set of $N$ commuting single-particle operators. This is equivalent for the $N$-particle state to be a product of single-particle eigenstates of the commuting set of single-particle operators. ${ }^{8}$ During the MF procedure, only product states are used as trial states, and thus, MF rotations are unitary operations that transform one product state into another. Having a multi-particle state to be a product of single-particle states can be seen as the origin for the term mean-field. Indeed, each particle is described by an eigenstate of some single-particle operator as if it does not interact with other particles but rather is in some effective potential or a mean-field created by other particles.
However, this consideration does not provide a straightforward path to formulating necessary and sufficient conditions for the Hamiltonian exact solvability by the MF procedure. In other words, what many-body Hamiltonians can have eigenstates that are products of one-particle states? There are well-known sufficient conditions for the MF-solvability: separable Hamiltonians, mainly appearing with distinguishable particles like qubits, and the Hamiltonians quadratic in fermionic and bosonic creation and annihilation operators. Yet, these cases do not cover all MF-solvable Hamiltonians. For example, it was found that for distinguishable particles there exist a large class of non-separable MF-solvable Hamiltonians. ${ }^{9,10}$

In this work, using a unifying Lie-algebraic framework we will provide necessary and sufficient conditions for the Hamiltonian to be MF-solvable for both distinguishable and indistinguishable particles. Similar Lie-algebraic consideration was done previously in Ref. 4 , but due to a different focus, Ref. 4 did not cover MF-solvable Hamiltonians that require multiple different unitary rotations to obtain all eigenstates (classes higher than 1 in our classification).

Our definition of MF-solvable Hamiltonians is based on the condition that all eigenstates of such Hamiltonians should be MF states. To define general MF states, we introduce a set of operators closed with respect to commutation, the Lie algebra, whose elements are sufficient for expressing the Hamiltonian. These Lie algebra operators constitute mathematical generalization of single-particle operators. The Lie algebra contains subsets of fully commuting operators, it is convenient to use one of the largest fully-commuting subsets or a Cartan sub-algebra (CSA). The MF states are defined as all states that can be constructed from eigenstates of a CSA by unitary transformations obtained using exponentiation of Lie algebra operators. Such MF states cover Slater determinants in the Hartree-Fock method ${ }^{1}$ and product states in qubit meanfield $^{11}$ and Hartree-Fock-Bogoliubov ${ }^{12}$ theories. These states can be also defined for an arbitrary Lie algebra that is chosen for expression of the Hamiltonian.

The main applicational value in the general formula- 
tion of the MF-solvability originates from advantages in changing one set of operators (forming a Lie algebra) to another in many-body Hamiltonians. It was found that MF theories can perform differently depending on the operator realizations for the same Hamiltonian. ${ }^{11}$ Moreover, there are examples of Hamiltonians that are not MF-solvable in one realization but are MF-solvable in another operator realization (e.g. $X Y$-model ${ }^{13}$ ). Also, with the progress in quantum hardware, it becomes necessary to reformulate various many-body Hamiltonians in terms of qubit operators ${ }^{7,14,15}$ and to be able to transfer many-body methods between different realizations.

The rest of the paper is organized as follows. Section II presents Lie-algebraic framework for quantum Hamiltonians, defines MF-solvable Hamiltonians of different classes, and comments on computational procedures identifying the MF-solvable Hamiltonians within the Hamiltonian of interest. Section III contains applications of the MF formalism for fermionic and qubit realizations of the electronic molecular Hamiltonian. Section IV concludes and provides further outlook.

\section{THEORY}

\section{A. Lie-algebraic framework}

Any Hamiltonian can be written as a polynomial expression of some elementary linear operators $\left\{\hat{A}_{k}\right\}$

$$
\hat{H}=\sum_{k} c_{k} \hat{A}_{k}+\sum_{k k^{\prime}} d_{k k^{\prime}} \hat{A}_{k} \hat{A}_{k^{\prime}}+\ldots,
$$

where $c_{k}$ and $d_{k k^{\prime}}$ are some constants. Choosing $\left\{\hat{A}_{k}\right\}$ appropriately allows one to have a set that is closed with respect to the commutation operation

$$
\left[\hat{A}_{i}, \hat{A}_{j}\right]=\sum_{k} \xi_{i j}^{(k)} \hat{A}_{k}
$$

where $\xi_{i j}^{(k)}$ are so-called structural constants from the number field $\mathbb{K}$, thus, $\left\{\hat{A}_{k}\right\}$ forms a Lie algebra, $\mathcal{A} .{ }^{16}$ Note that products like $\hat{A}_{k} \hat{A}_{k^{\prime}}$ and higher powers of $\mathcal{A}$ elements do not generally belong to the Lie algebra $\mathcal{A}$, instead they are part of a universal enveloping algebra (UEA), $\mathcal{E}_{\mathcal{A}}$, which is built as a direct sum of tensor powers of the Lie algebra

$$
\mathcal{E}_{\mathcal{A}}=\mathbb{K} \oplus \mathcal{A} \oplus(\mathcal{A} \otimes \mathcal{A}) \oplus \ldots,
$$

where the Lie bracket operation is equivalent to the commutator. Thus, any Hamiltonian is an element of some UEA.

Among various Lie algebras that can be chosen for realization of $\hat{H}$, for our purpose, it will be convenient to use reductive Lie algebras as $\mathcal{A}$. There are two reasons for this choice. First, a reductive algebra is a direct sum of abelian and semisimple Lie algebras, and structural theories for both of these algebra types are well-understood. ${ }^{17}$
Second, in Appendix A we show that field $\mathbb{K}$ can be chosen to be real for $\mathcal{A}$ realizing $\hat{H}$, and thus, $\mathcal{A}$ is a compact Lie algebra, which allows us to use some powerful theorems for this type of algebras. Since the compact Lie algebras are always reductive, Appendix A also shows that it is possible to realize the Hamiltonian using reductive Lie algebras.

Any reductive Lie algebra $\mathcal{A}$ has a maximal abelian sub-algebra $\mathcal{C} \subset \mathcal{A}$ that is referred to as the CSA. If $\mathcal{A}$ is a direct sum of abelian $\mathfrak{a}$ and semisimple $\mathfrak{s}$ algebras, then $\mathcal{C}$ will be a direct sum of $\mathfrak{a}$ and a CSA of $\mathfrak{s}$. The latter is a maximal abelian sub-algebra of $\mathfrak{s}$ whose elements are ad-diagonalizable. ${ }^{17}$ We will denote elements of $\mathcal{C}$ as $\hat{C}_{k}$ 's. The UEA constructed from $\mathcal{C}$, $\mathcal{E}_{\mathcal{C}}$, is abelian as well. Thus, in principle, all elements of $\mathcal{E}_{\mathcal{C}}$ have a common set of eigenfunctions, $\left|C_{1}, \ldots C_{N}\right\rangle$ : $\hat{C}_{k}\left|C_{1}, \ldots C_{N}\right\rangle=C_{k}\left|C_{1}, \ldots C_{N}\right\rangle$. These eigenfunctions can be used to construct functional spaces for representation of $\mathcal{A}$ and operators from $\mathcal{E}_{\mathcal{A}}$.

In all Lie algebras that are used for expressing manybody problems, common eigenstates of $\mathcal{C}$ are relatively easy to obtain. Therefore, solving the eigenvalue problem for the Hamiltonian in Eq. (1) can be done by finding a unitary operator $\hat{U}$ that transforms $\hat{H}$ into a polynomial over the CSA elements

$$
\hat{U} \hat{H} \hat{U}^{\dagger}=\sum_{k} c_{k} \hat{C}_{k}+\sum_{k k^{\prime}} d_{k k^{\prime}} \hat{C}_{k} \hat{C}_{k^{\prime}}+\ldots
$$

Hamiltonians are hermitian operators, which makes them diagonalizable. Therefore, $\hat{U}$ always exist since Eq. (4) provides such a diagonal form of the Hamiltonian in the basis of the CSA eigen-states. The difficulty is that for a general Hamiltonian, $\hat{U}$ is an element of the Lie group corresponding to the exponential map of the exponentially large Lie algebra obtained by commutator closure of the UEA. Thus, it can be very difficult to find such $\hat{U}$ in general.

\section{B. Mean-field solvable Hamiltonians}

To define a set of the MF solvable Hamiltonians we introduce a restricted class of unitary transformations, MF rotations

$$
\hat{U}_{\mathrm{MF}}=\prod_{k=1}^{|\mathcal{A}|} e^{\theta_{k} \hat{A}_{k}}
$$

where $\theta_{k}$ are parameters defining MF rotational angles, $\hat{A}_{k}$ are anti-hermitian operators, and $|\mathcal{A}|$ is the number of $\hat{A}_{k}$ generators in $\mathcal{A}$. $\hat{U}_{\mathrm{MF}}$ 's form the universal covering Lie group corresponding to the Lie algebra $\mathcal{A}$. In spite of general non-commutativity of generators $\hat{A}_{k}$, due to the algebraic closure according to Eq. (2), any pair of $\hat{U}_{\mathrm{MF}}$ 's that are different by the order of exponents in Eq. (5) can be made equal by selecting $\theta_{k}$ 's in one of these $\hat{U}_{\mathrm{MF}}$ 's. ${ }^{18}$ Another property of the MF unitaries that stems from 
the algebraic closure is

$$
\hat{U}_{\mathrm{MF}}^{\dagger} \hat{A}_{k^{\prime}} \hat{U}_{\mathrm{MF}}=\sum_{k} c_{k} \hat{A}_{k}
$$

where $\hat{A}_{k^{\prime}}$ is any Lie algebra element. This property is essential for computational advantage of MF theories since it results in preservation of the degree for any polynomial function of algebra elements $\hat{A}_{k}$ upon transformation by $\hat{U}_{\mathrm{MF}}$.

We define the mean-field solvable Hamiltonians as the ones that have the form

$$
\hat{H}_{\mathrm{MF}}=\sum_{J} \hat{V}_{J}\left|\bar{C}_{J}\right\rangle E_{J}\left\langle\bar{C}_{J}\right| \hat{V}_{J}^{\dagger},
$$

where $E_{J}$ are eigen-values, $\left|\bar{C}_{J}\right\rangle=\left|C_{1}^{(J)}, \ldots C_{N}^{(J)}\right\rangle$ are basis states that are eigen-states of the CSA operators, and $\hat{V}_{J}$ are unitary MF rotations (Eq. (5)). Here, any basis state $\left|\bar{C}_{J}\right\rangle$ can be transformed to the eigenstate of $\hat{H}_{\mathrm{MF}}$ using the MF rotation $\hat{U}_{\mathrm{MF}}=\hat{V}_{J}$. It is convenient to separate Eq. (7) Hamiltonians into classes, class $K$ Hamiltonians contain $K$ different unitary transformations $\left\{\hat{V}_{J}\right\}_{J=1}^{K}$. For all classes, due to hermiticity of $\hat{H}_{\mathrm{MF}}$, there is the orthogonality condition

$$
\left\langle\bar{C}_{J}\left|\hat{V}_{J}^{\dagger} \hat{V}_{I}\right| \bar{C}_{I}\right\rangle=\delta_{J I}
$$

The form given by Eq. (7) is sufficient for the MFsolvability because all its eigenstates can be obtained using the MF rotations. It is also a necessary condition because if a hermitian Hamiltonian has eigenstates in the MF form $\hat{V}_{J}\left|\bar{C}_{J}\right\rangle$, then it can be always written as Eq. (7) with the condition that all eigenstates are orthogonal. The main shortcoming of Eq. (7) as a criterion for determining the MF-solvability of an arbitrary Hamiltonian is that it does not employ only the elements of the Lie algebra but also requires projections $\left|\bar{C}_{J}\right\rangle\left\langle\bar{C}_{J}\right|$ and the orthogonality condition (Eq. (8)). In what follows we will express projectors as functions of CSA elements and introduce the necessary orthogonality with minimal constraints on the MF unitaries. Fully algebraic definitions of MF-solvable Hamiltonians will be provided for each class separately.

Class 1: This class corresponds to a single unitary transformation $\hat{V}_{J}=\hat{U}_{\mathrm{MF}}^{\dagger}$, hence Eq. (7) can be written as

$$
\hat{H}_{\mathrm{MF}, 1}=\hat{U}_{\mathrm{MF}}^{\dagger} \sum_{J}\left|\bar{C}_{J}\right\rangle E_{J}\left\langle\bar{C}_{J}\right| \hat{U}_{\mathrm{MF}}
$$

The sum over $J$ can be seen as an arbitrary operator in the eigen-subspace of the CSA, therefore it can be also written as a general Taylor series over the CSA elements

$$
\begin{aligned}
\sum_{J}\left|\bar{C}_{J}\right\rangle E_{J}\left\langle\bar{C}_{J}\right| & =\sum_{k} c_{k} \hat{C}_{k}+\sum_{k k^{\prime}} d_{k k^{\prime}} \hat{C}_{k} \hat{C}_{k^{\prime}}+\ldots \\
& =F\left(\hat{C}_{k}\right) .
\end{aligned}
$$

Thus, by substituting the $J$-sum in Eq. (9) by the Taylor expansion, we obtain

$$
\hat{H}_{\mathrm{MF}, 1}=\hat{U}_{\mathrm{MF}}^{\dagger} F\left(\hat{C}_{k}\right) \hat{U}_{\mathrm{MF}},
$$

hence, Eq. (12) is the algebraic form equivalent to Eq. (7) for class 1 .

Class 2: According to Eq. (7), for class 2, there are two MF unitary transformations $\hat{V}_{1}$ and $\hat{V}_{2}$ that create two sets of eigen-states by acting on two groups of basis states, $\left\{\left|\bar{C}_{J_{1}}\right\rangle\right\}$ and $\left\{\left|\bar{C}_{J_{2}}\right\rangle\right\}$. Let us introduce projectors on subspaces $\left\{\left|\bar{C}_{J_{1}}\right\rangle\right\}$ and $\left\{\left|\bar{C}_{J_{2}}\right\rangle\right\}$

$$
\hat{P}_{1}=\sum_{J_{1}}\left|\bar{C}_{J_{1}}\right\rangle\left\langle\bar{C}_{J_{1}}\right|
$$

and $\hat{P}_{1}^{\perp}=1-\hat{P}_{1}$. Individual projectors $\left|\bar{C}_{J_{1}}\right\rangle\left\langle\bar{C}_{J_{1}}\right|$ can be written in the algebraic form using Löwdin's projection formula

$$
\left|\bar{C}_{J_{1}}\right\rangle\left\langle\bar{C}_{J_{1}}\right|=\prod_{k} \prod_{C_{k} \neq C_{k}^{\left(J_{1}\right)}} \frac{\hat{C}_{k}-C_{k}}{C_{k}^{\left(J_{1}\right)}-C_{k}} .
$$

The introduced projectors allow us to formulate the algebraic form of the class-2 MF-solvable Hamiltonians

$$
\hat{H}_{\mathrm{MF}, 2}=\hat{U}_{1}^{\dagger}\left(F_{1}\left(\hat{C}_{k}\right) \hat{P}_{1}+U_{2}^{\dagger} F_{2}\left(\hat{C}_{k}\right) \hat{U}_{2} \hat{P}_{1}^{\perp}\right) \hat{U}_{1},
$$

where $\left\{\hat{U}_{i}\right\}_{i=1,2}$ are MF unitaries and $\left\{F_{i}\left(\hat{C}_{k}\right)\right\}_{i=1,2}$ are analytic functions of CSA elements. Here, based on $\hat{C}_{k}$ eigenvalues after the $\hat{U}_{1}$ transformation, all states are partitioned in two subspaces $\left\{\left|\bar{C}_{J_{1}}\right\rangle\right\}$ and $\left\{\left|\bar{C}_{J_{2}}\right\rangle\right\}$. The states from the first subspace acquire their eigenvalues according to the $F_{1}\left(\hat{C}_{k}\right)$ function, and the states from the second subspace rotated with $\hat{U}_{2}$ so that the eigenvalues of the rotated states would be determined by $F_{2}\left(\hat{C}_{k}\right)$.

Hamiltonian $\hat{H}_{\mathrm{MF}, 2}$ is hermitian because $F_{1}\left(\hat{C}_{k}\right)$ and $\hat{U}_{2}^{\dagger} F_{2}\left(\hat{C}_{k}\right) \hat{U}_{2}$ commute with $\hat{P}_{1}$ and $\hat{P}_{1}^{\perp}$. The commutation of $\hat{P}_{1}^{\perp}$ with $\hat{U}_{2}^{\dagger} F_{2}\left(\hat{C}_{k}\right) \hat{U}_{2}$ is not satisfied by a general $\hat{U}_{2}$ transformation, and thus it introduces a constraint on the $\hat{U}_{2}$ transformation. To satisfy this constraint it is sufficient to remove $\hat{A}_{k}$ 's in $\hat{U}_{2}$ that do not commute with $\hat{P}_{1}^{\perp}$.

To prove that Eq. (15) can be a criterion for the class-2 MF-solvability, we show that it is equivalent to Eq. (7) for class 2. Two unitaries of Eq. (15), $\hat{U}_{1}$ and $\hat{U}_{2}$, should be used as $\hat{U}_{1}^{\dagger}$ and $\left(\hat{U}_{2} \hat{U}_{1}\right)^{\dagger}$ to obtain all eigenstates. Thus, we associate $\hat{V}_{1}$ with $\hat{U}_{1}^{\dagger}$ and $\hat{V}_{2}$ with $\left(\hat{U}_{2} \hat{U}_{1}\right)^{\dagger}$, then $\hat{U}_{2}=$ $\hat{V}_{2}^{\dagger} \hat{V}_{1}$. The commutativity of $\hat{U}_{2}$ with the $\hat{P}_{1}$ projector is necessary to maintain the orthogonality between the $\left\{\left|\bar{C}_{J_{1}}\right\rangle\right\}$ and $\left\{\left|\bar{C}_{J_{2}}\right\rangle\right\}$ subspaces

$$
\begin{aligned}
\left\langle\bar{C}_{J_{1}}\left|\hat{V}_{1}^{\dagger} \hat{V}_{2}\right| \bar{C}_{J_{2}}\right\rangle & =\left\langle\bar{C}_{J_{1}}\left|\hat{U}_{1} \hat{U}_{1}^{\dagger} \hat{U}_{2}^{\dagger}\right| \bar{C}_{J_{2}}\right\rangle \\
& =\left\langle\bar{C}_{J_{1}}\left|\hat{U}_{2}^{\dagger}\right| \bar{C}_{J_{2}}\right\rangle=\left\langle\bar{C}_{J_{1}} \mid \bar{C}_{J_{2}}\right\rangle=0 .
\end{aligned}
$$

Indeed, orthogonal subspaces $\left\{\left|\bar{C}_{J_{1}}\right\rangle\right\}$ and $\left\{\left|\bar{C}_{J_{2}}\right\rangle\right\}$ correspond to the $\hat{P}_{1}$ and $\hat{P}_{1}^{\perp}$ projectors, hence, commutativity $\left[\hat{U}_{2}, \hat{P}_{1}\right]=0$ is necessary and sufficient for $\hat{U}_{2}^{\dagger}$ to have $\left\{\left|\bar{C}_{J_{2}}\right\rangle\right\}$ as an invariant subspace, and thus not to disrupt the orthogonality.

Higher classes: Further generalizations to higher classes can be done recursively. For class 3, the subspace $\left\{\left|\bar{C}_{J_{2}}\right\rangle\right\}$ needs to be partitioned further onto two 
subspaces with corresponding projectors $\hat{P}_{2}$ and $\hat{P}_{2}^{\perp}$. The algebraic form of class-3 MF-solvable Hamiltonian is obtained from Eq. (15) using the substitution

$$
F_{2}\left(\hat{C}_{k}\right) \rightarrow F_{2}\left(\hat{C}_{k}\right) \hat{P}_{2}+\hat{U}_{3}^{\dagger} F_{3}\left(\hat{C}_{k}\right) \hat{U}_{3} \hat{P}_{2}^{\perp},
$$

where $\hat{U}_{3}$ is a new MF transformation commuting with $\hat{P}_{2}^{\perp}, F_{3}$ is an analytic function of CSA elements, and $\hat{P}_{2}^{\perp}$ projects out the states that can be obtained by the $\left(\hat{U}_{2} \hat{U}_{1}\right)^{\dagger}$ transformation.

Proving the equivalence of algebraic forms of the MFsolvable Hamiltonians of higher classes (e.g. Eq. (16)) to Eq. (7) can be done by straightforward extension of the arguments given for class 2 . Thus, as the criterion for the MF-solvability, one can use either Eq. (7) with the extra orthogonality condition in Eq. (8) or algebraic expressions (e.g. Eqs. (12), (15)) whose form depends on the class and where the orthogonality condition is substituted by the commutativity requirement between projectors and unitary transformations.

\section{Computational aspect}

Using a particular Lie algebra it is easy to construct an MF-solvable Hamiltonian of any class. The problem of identifying whether a particular Hamiltonian is MFsolvable, and what its class is, can be efficiently addressed by finding its eigenstates using the variational approach. The only caveat is that it is necessary to find all MF solutions of a given Hamiltonian, since it is possible to envision the Hamiltonian that has only a few MF solutions and non-MF states for the rest of the spectrum. Appendix B presents an example of the Hamiltonian that is only partially solvable by the MF procedure and discusses a procedure for establishing partial or full MF-solvability. If the Hamiltonian is realized using only lower powers of the corresponding UEA (e.g., two-electron Hamiltonians) then the algorithm for establishing MF-solvability and finding all MF solutions requires a non-linear optimization with a polynomial number of parameters with the number of particles. This makes low-degree MF-solvable Hamiltonians classically tractable.

In the case when it is known that the Hamiltonian is MF-solvable, finding the lowest eigenstate and its energy is equivalent to minimization of the Hamiltonian energy functional with respect to $\theta_{k}$ parameters in Eq. (5). For a non-degenerate ground state, the MF solvability guarantees that the energy function has only one global minimum corresponding to the ground state. All other states correspond to saddle points in the energy function, and thus, common optimization procedures will usually converge to the ground state in this case.

Another practically interesting question is related to decomposition of a given Hamiltonian in terms of MFsolvable Hamiltonians. This problem was addressed in Ref. 3 for class 1, where it was shown that if the Hamiltonian contains only a few lower powers of $\mathcal{A}$ elements in Eq. (1), the decomposition procedure can be done in polynomial efforts with respect to the size of $\mathcal{A}$. The decomposition with higher classes has not been addressed yet, and it appears to be an exponentially hard problem in general because the projectors in Eqs. (15) and (16) can contain high powers of CSA elements. Polynomial scaling of such decompositions is only possible if the overall power of $\hat{A}_{k}$ elements in Eq. (1) is restricted.

\section{APPLICATIONS}

Here we will illustrate how the Lie-algebraic framework for identifying the MF-solvable Hamiltonians is applicable to a few commonly used realizations for the electronic Hamiltonian. Historically, all these realizations had their MF procedures developed from the quasi-particle (Fermi liquid) types of considerations. These considerations can be related to Lie algebras, but the relation is not obvious and will be further clarified.

Besides illustration of MF-solvable Hamiltonians, we will use well-developed structural theory for reductive Lie algebras to produce standard ladder operators, which provide a simple way to generate excited states starting from the ground state. For any reductive Lie algebra, there are only two types of operators, the ones that form the CSA $\left\{\hat{C}_{k}\right\}$ and the ladder operators $\left\{\hat{L}_{j}^{ \pm}\right\}$

$$
\left[\hat{C}_{k}, \hat{L}_{j}^{ \pm}\right]= \pm \alpha_{j k} \hat{L}_{j}^{ \pm}
$$

where $\alpha_{j k}$ are constants. $\left\{\hat{L}_{j}^{ \pm}\right\}$allow one to generate the entire set of eigenstates starting from a maximum (minimum) weight state by using lowering (raising) ladder operators. The only necessary condition for generating the ladder operators is to consider the complex extension of the original compact Lie algebra, which is achieved by extending $\mathbb{K}$ to $\mathbb{C}$.

\section{A. Fermionic algebras}

The electronic Hamiltonian $\hat{H}_{e}$, starting from its second quantized form can be realized using the elements of different Lie algebras. For example, the common second quantized form of $\hat{H}_{e}$ is

$$
\hat{H}_{e}=\sum_{p q} h_{p q} \hat{a}_{p}^{\dagger} \hat{a}_{q}+\sum_{p q r s} g_{p q, r s} \hat{a}_{p}^{\dagger} \hat{a}_{q}^{\dagger} \hat{a}_{r} \hat{a}_{s}
$$

where $h_{p q}$ and $g_{p q, r s}$ are real constants. A set of $2 N$ $\hat{a}_{p}^{\dagger}, \hat{a}_{q}$ operators, where $N$ is the number of spin-orbitals, is not closed with respect to commutation

$$
\begin{aligned}
& {\left[\hat{a}_{p}^{\dagger}, \hat{a}_{q}^{\dagger}\right]=2 \hat{a}_{p}^{\dagger} \hat{a}_{q}^{\dagger}} \\
& {\left[\hat{a}_{p}, \hat{a}_{q}\right]=2 \hat{a}_{p} \hat{a}_{q}} \\
& {\left[\hat{a}_{p}^{\dagger}, \hat{a}_{q}\right]=2 \hat{a}_{p}^{\dagger} \hat{a}_{q}-\delta_{p q} .}
\end{aligned}
$$

To transform this set to a Lie algebra one needs to add all possible products $\hat{E}^{p q}=\hat{a}_{p}^{\dagger} \hat{a}_{q}^{\dagger}, \hat{E}_{p q}=\hat{a}_{p} \hat{a}_{q}$, and $\hat{E}_{q}^{p}=$ 
$\hat{a}_{p}^{\dagger} \hat{a}_{q}$. This addition provides the following commutation relation illustrating the algebraic closure

$$
\begin{aligned}
{\left[\hat{E}_{q}^{p}, \hat{E}_{s}^{r}\right] } & =\hat{E}_{s}^{p} \delta_{q r}-\hat{E}_{q}^{r} \delta_{p s} \\
{\left[\hat{E}_{q}^{p}, \hat{E}_{r s}\right] } & =\hat{E}_{q r} \delta_{p s}-\hat{E}_{q s} \delta_{p r} \\
{\left[\hat{E}^{p q}, \hat{E}_{r s}\right] } & =\hat{E}_{r}^{q} \delta_{p s}+\hat{E}_{s}^{p} \delta_{q r}-\hat{E}_{s}^{q} \delta_{p r}-\hat{E}_{r}^{p} \delta_{q s} \\
{\left[\hat{E}_{p q}, \hat{E}_{r s}\right] } & =\left[\hat{E}^{p q}, \hat{E}^{r s}\right]=0
\end{aligned}
$$

and

$$
\begin{aligned}
{\left[\hat{a}_{p}, \hat{E}_{s}^{r}\right] } & =\delta_{p r} \hat{a}_{s} \\
{\left[\hat{a}_{p}, \hat{E}_{r s}\right] } & =\left[\hat{a}_{p}^{\dagger}, \hat{E}^{r s}\right]=0 \\
{\left[\hat{a}_{p}, \hat{E}^{r s}\right] } & =\delta_{p r} \hat{a}_{s}^{\dagger}-\delta_{p s} \hat{a}_{r}^{\dagger} .
\end{aligned}
$$

The operators $\left\{\hat{a}_{p}^{\dagger}, \hat{a}_{p}, \hat{E}_{q}^{p}, \hat{E}^{p q}, \hat{E}_{p q}\right\}$ are not standard generators of the compact $\mathfrak{s o}(2 N+1)$ Lie algebra, but their linear combinations are sufficient for constructing these generators. ${ }^{19,20}$ This construction is facilitated by introducing the Majorana operators

$$
\begin{aligned}
\hat{\gamma}_{2 p-1} & =i\left(\hat{a}_{p}-\hat{a}_{p}^{\dagger}\right) / \sqrt{2} \\
\hat{\gamma}_{2 p} & =\left(\hat{a}_{p}^{\dagger}+\hat{a}_{p}\right) / \sqrt{2},
\end{aligned}
$$

which allow us to introduce the $\mathfrak{s o}(2 N+1)$ generators as

$$
\begin{aligned}
& \hat{S}_{j 0}=-\hat{S}_{0 j}=-i \gamma_{j}, \quad(j=1, \ldots 2 N) \\
& \hat{S}_{j k}=\left[\gamma_{j}, \gamma_{k}\right] / 4=\gamma_{j} \gamma_{k} / 2(j \neq k ; j, k=1, \ldots 2 N) .
\end{aligned}
$$

Generators $\hat{S}_{j k}(j, k=0,1 \ldots, 2 N)$ satisfy regular $\mathfrak{s o}(2 N+$ 1) commutation relations

$$
\left[\hat{S}_{i j}, \hat{S}_{k l}\right]=\delta_{j k} \hat{S}_{i l}+\delta_{i l} \hat{S}_{j k}-\delta_{i k} \hat{S}_{j l}-\delta_{j l} \hat{S}_{i k}
$$

There are $2 N(2 N+1) / 2=2 N^{2}+N$ of $\hat{S}_{j k}$ operators in total.

The $\mathfrak{s o}(2 N+1)$ algebra contains smaller sub-algebras that can be used to realize the Hamiltonian: $\mathfrak{s o}(2 N)$ and $\mathfrak{u}(N) .{ }^{19} \mathfrak{s o}(2 N)$ requires only $\left\{\hat{E}_{q}^{p}, \hat{E}^{p q}, \hat{E}_{p q}\right\}$ operators which can form $\hat{S}_{j k}(j, k=1 \ldots, 2 N)$ generators and give rise to the following realization of the Hamiltonian

$$
\hat{H}_{e, 2}=\sum_{p q} h_{p q} \hat{E}_{q}^{p}+\sum_{p q r s} g_{p q, r s} \hat{E}^{p q} \hat{E}_{r s} .
$$

Lie algebra $\mathfrak{u}(N)$ realization is obtained using the following transformations:

$$
\begin{aligned}
\hat{H}_{e, 3} & =\sum_{p q} h_{p q} \hat{a}_{p}^{\dagger} \hat{a}_{q}+\sum_{p q r s} g_{p q, r s}\left(\hat{a}_{p}^{\dagger} \hat{a}_{s} \delta_{r q}-\hat{a}_{p}^{\dagger} \hat{a}_{r} \hat{a}_{q}^{\dagger} \hat{a}_{s}\right) \\
& =\sum_{p q}\left[h_{p q}+\sum_{r} g_{p r, r q}\right] \hat{a}_{p}^{\dagger} \hat{a}_{q} \\
& -\sum_{p q r s} g_{p q, r s} \hat{a}_{p}^{\dagger} \hat{a}_{r} \hat{a}_{q}^{\dagger} \hat{a}_{s} \\
& =\sum_{p q} \tilde{h}_{p q} \hat{E}_{q}^{p}-\sum_{p q r s} g_{p q, r s} \hat{E}_{r}^{p} \hat{E}_{s}^{q} .
\end{aligned}
$$

$\left\{\hat{E}_{q}^{p}\right\}$ operators can be transformed into the $\mathfrak{u}(N)$ compact generators

$$
\begin{aligned}
& \hat{\kappa}_{p q}=\left(\hat{E}_{q}^{p}-\hat{E}_{p}^{q}\right) / 2 \\
& \hat{\kappa}_{p q}^{\prime}=i\left(\hat{E}_{q}^{p}+\hat{E}_{p}^{q}\right) / 2 .
\end{aligned}
$$

\section{1. $\mathfrak{u}(N)$ algebra}

Lie algebra $\mathfrak{u}(N)$ can be decomposed into direct sum of $\mathfrak{u}(1)$ and $\mathfrak{s u}(N)$. A $\mathfrak{u}(1)$ generator commutes with all elements of $\mathfrak{u}(N)$ and can be expressed as $i \hat{N}=i \sum_{p} \hat{E}_{p}^{p}$. The CSA for $\mathfrak{u}(N)$ is $N$ elements $i \hat{E}_{p}^{p}$, which are antihermitian versions of the usual spin-orbital occupation operators. The ladder operators can be defined as $\hat{L}_{q}^{+}=$ $\hat{E}_{p}^{q}$ and $\hat{L}_{q}^{-}=\hat{E}_{q}^{p}$ with $\alpha_{q p}=1$ in Eq. (17). Note that for the complex extension of $\mathfrak{u}(\mathfrak{n})$ one can take $\hat{C}_{p}=\hat{E}_{p}^{p}$ to avoid extra imaginary units in definitions.

The components of MF-solvable Hamiltonians in this algebra are one-particle unitary transformations

$$
\hat{U}=\prod_{p \neq q} e^{\hat{\kappa}_{p q} \theta_{p q}} e^{\hat{\kappa}_{p q}^{\prime} \phi_{p q}}
$$

and the CSA elements $\hat{C}_{p}=i \hat{E}_{p}^{p}$. Appendix $\mathrm{C}$ presents two nontrivial (non-one-electron) $\mathfrak{u}(N) \mathrm{MF}-$ solvable Hamiltonians of class 1 and 2.

$$
\text { 2. } \mathfrak{s o}(2 N) \text { and } \mathfrak{s o}(2 N+1) \text { algebras }
$$

The CSA for $\mathfrak{s o}(2 N)$ and $\mathfrak{s o}(2 N+1)$ is the same and contains any $N$ elements with non-overlapping indices, for example $\mathcal{C}_{\mathcal{S}}=\left\{\hat{S}_{12}, \hat{S}_{34}, \ldots . \hat{S}_{(2 N-1) 2 N}\right\}$. Addition of any $\hat{S}_{0 j}$ element is not feasible due to unavoidable index overlap. $\mathcal{C}_{\mathcal{S}}$ can be connected with the $\mathfrak{u}(N)$ CSA by expressing the elements in $\hat{a}_{p}^{\dagger}$ and $\hat{a}_{q}$ operators:

$$
\begin{aligned}
\hat{S}_{(2 p-1) 2 p} & =\frac{1}{2} \hat{\gamma}_{2 p-1} \hat{\gamma}_{2 p}=\frac{i}{4}\left(\hat{a}_{p}-\hat{a}_{p}^{\dagger}\right)\left(\hat{a}_{p}^{\dagger}+\hat{a}_{p}\right) \\
& =\frac{i}{4}\left(1-2 \hat{E}_{p}^{p}\right) .
\end{aligned}
$$

For the complex extension of the $\mathfrak{s o}$ algebras, it is possible to use $\hat{L}_{p}^{ \pm}$obtained for $\mathfrak{u}(N)$, but there are also additional ladder operators with $\hat{E}_{p q}\left(\hat{E}^{q p}\right)$

$$
\begin{gathered}
{\left[\hat{E}_{p}^{p}, \hat{E}_{p q}\right]=-\hat{E}_{p q}, \quad\left[\hat{E}_{q}^{q}, \hat{E}_{p q}\right]=-\hat{E}_{p q}} \\
{\left[\hat{E}_{p}^{p}, \hat{E}^{q p}\right]=\hat{E}^{q p}, \quad\left[\hat{E}_{p}^{p}, \hat{E}^{q p}\right]=\hat{E}^{q p}}
\end{gathered}
$$

and $\hat{a}_{p}\left(\hat{a}_{p}^{\dagger}\right)$ for $\mathfrak{s o}(2 N+1)$ only

$$
\begin{aligned}
& {\left[\hat{E}_{p}^{p}, \hat{a}_{p}\right]=-\hat{a}_{p}} \\
& {\left[\hat{E}_{p}^{p}, \hat{a}_{p}^{\dagger}\right]=\hat{a}_{p}^{\dagger} .}
\end{aligned}
$$

Another popular set of CSAs that can be built here, are those that do not conserve the number of particles. Using the Hartree-Fock-Bogoliubov ${ }^{12}$ unitary transformation conserving the anti-commutation relations of $\hat{a}_{p}^{\dagger}$ and $\hat{a}_{q}$ operators, one can introduce linear combinations

$$
\begin{aligned}
& \hat{\beta}_{q}^{\dagger}=\sum_{p} U_{p q} \hat{a}_{p}^{\dagger}+V_{p q} \hat{a}_{p} \\
& \hat{\beta}_{q}=\sum_{p} V_{p q}^{*} \hat{a}_{p}^{\dagger}+U_{p q}^{*} \hat{a}_{p},
\end{aligned}
$$


where $U$ and $V$ matrices satisfying the following conditions:

$$
\begin{aligned}
U^{\dagger} U+V^{\dagger} V & =1 \\
U U^{\dagger}+V^{*} V^{T} & =1 \\
U^{T} V+V^{T} U & =0 \\
U V^{\dagger}+V^{*} U^{T} & =0 .
\end{aligned}
$$

A set of $\left\{i \hat{\beta}_{p}^{\dagger} \hat{\beta}_{p}\right\}$ forms a CSA for the $\mathfrak{s o}(2 N)$ and $\mathfrak{s o}(2 N+1)$ algebras, while quadratic and linear operators constructed out of $\hat{\beta}_{p}^{\dagger}$ and $\hat{\beta}_{q}$ form the ladder operators analogously to the same construction with $\hat{a}_{p}^{\dagger}$ and $\hat{a}_{q}$ operators.

\section{General connection between Clifford and Lie algebras via Majorana operators}

Majorana operators are convenient because they easily connect quasi-particle-like pictures and Lie-algebraic frameworks. Any set of quasi-particle fermionic operators $\left\{\hat{a}_{p}^{\dagger}\right\}$ or $\left\{\hat{a}_{p}\right\}$ form Grassmann algebras: $\left\{\hat{a}_{p}^{\dagger}, \hat{a}_{q}^{\dagger}\right\}=0$ and $\left\{\hat{a}_{p}, \hat{a}_{q}\right\}=0$, but combinations of these sets do not, since $\left\{\hat{a}_{p}, \hat{a}_{q}^{\dagger}\right\}=\delta_{p q}$. On the other hand, Majorana operators make proper combinations of these sets to form a Clifford algebra: $\left\{\hat{\gamma}_{p}, \hat{\gamma}_{q}\right\}=\delta_{p q}$. Clifford algebras with $M$ generators always produce $\mathfrak{s o}(M+1)$ Lie algebras from the commutator closure, this process was illustrated in Eqs. (31) and (32). The CSA of the resulting $\mathfrak{s o}(M+1)$ algebra can always be selected in the part which is quadratic in Majorana operators, and this part forms the $\mathfrak{s o}(M)$ algebra. One can always organize the quasi-particle operators (or ladder operators) using a linear combinations of Majorana operators that return back to $\hat{a}_{p}^{\dagger}$ - and $\hat{a}_{q}$-like operators. These operators can be rotated by the quadratic $(\mathfrak{s o}(M))$ elements

$$
e^{\theta \hat{\gamma}_{q} \hat{\gamma}_{r}} \hat{\gamma}_{p} e^{-\theta \hat{\gamma}_{q} \hat{\gamma}_{r}}=\sum_{s} c_{s}(\theta) \hat{\gamma}_{s}
$$

into some linear combinations of linear Majorana terms. This is based on the commutation relation $\left[\hat{\gamma}_{p}, \hat{\gamma}_{q} \hat{\gamma}_{r}\right]=$ $2\left(\hat{\gamma}_{r} \delta_{p q}-\hat{\gamma}_{q} \delta_{p r}\right)$, which guarantees production of linear terms. Thus, mean-field type of theories can be easily built if one has $M$ Clifford algebra elements, and it will guarantee existence of the $\mathfrak{s o}(M+1)$ Lie algebra with $\lfloor M / 2\rfloor$ CSA elements. Similar analysis was done in Ref. 21 using graph theory techniques and qubit representation for the Hamiltonian.

\section{B. Qubit algebras}

Another class of realizations of the electronic Hamiltonian can be obtained by mapping fermionic operators to qubits using the Jordan-Wigner, Bravyi-Kitaev, or similar fermionic-qubit mappings. ${ }^{6,7,15,22,23}$ Here, we will use the Jordan-Wigner mapping as the simplest for illustrative purpose

$$
\begin{aligned}
& \hat{a}_{p}=\left(\hat{x}_{p}-i \hat{y}_{p}\right) \otimes \hat{z}_{p-1} \otimes \hat{z}_{p-2} \ldots \otimes \hat{z}_{1} \\
& \hat{a}_{p}^{\dagger}=\left(\hat{x}_{p}+i \hat{y}_{p}\right) \otimes \hat{z}_{p-1} \otimes \hat{z}_{p-2} \ldots \otimes \hat{z}_{1} .
\end{aligned}
$$

This mapping produces

$$
\begin{gathered}
\hat{H}_{q}=\sum_{k} c_{k} \hat{P}_{k}, \\
\hat{P}_{k}=\otimes_{k=1}^{N} \hat{\sigma}_{k}
\end{gathered}
$$

where $c_{k}$ are numerical constants, and $\hat{\sigma}_{k}$ are either Pauli spin operators $\hat{x}_{k}, \hat{y}_{k}, \hat{z}_{k}$ or the identity $\hat{1}_{k}$. We can consider $\hat{P}_{k}$ 's as elements of the UEA where the Lie algebra is a direct sum of $N \mathfrak{s u}(2)$ 's: $\mathcal{S}=\mathfrak{s u}(2) \oplus \ldots \oplus \mathfrak{s u}(2)$ and $\mathbb{K}=\mathbb{R}$. $\mathcal{S}$ is a semi-simple Lie algebra with $3 N$ generators $\left(i \hat{x}_{k}, i \hat{y}_{k}, i \hat{z}_{k}\right)$.

There are $3^{N}$ CSAs for $\mathcal{S}$, which are based on selecting a particular Pauli operator $(\hat{x}, \hat{y}$ or $\hat{z})$ for each qubit and thus containing $N$ elements each, for example, $\left\{i \hat{z}_{k}\right\}_{k=1}^{N}$.

\section{Class-1 qubit mean-field Hamiltonians}

If one restricts unitaries to products of single-qubit operators

$$
\hat{U}_{\mathrm{QMF}}=\prod_{k=1}^{N} e^{i \tau_{k}\left(\bar{n}_{k}, \bar{\sigma}_{k}\right)},
$$

where $\tau_{k}$ is an amplitude, $\bar{n}_{k}$ is a unit vector on the Bloch sphere, and $\bar{\sigma}_{k}=\left(\hat{x}_{k}, \hat{y}_{k}, \hat{z}_{k}\right)$. The MF-solvable Hamiltonians within the qubit mean-field (QMF) approach ${ }^{11}$ are in the form of Eq. (12) with $\hat{U}_{\mathrm{MF}}=\hat{U}_{\mathrm{QMF}}$ and $\hat{C}_{k}=i \hat{z}_{k}$ (or any other CSA of $\mathcal{S}$ ). One-qubit rotations in the qubit space do not translate to one-electron fermionic transformations (Eq. (38)) as was shown. ${ }^{11}$ Therefore, the QMFsolvable Hamiltonians are different from those in the $\mathfrak{u}(N)$ algebra.

Ladder operators of $\mathcal{S}$ are constructed as in the complex extension of the $\mathfrak{s u}(2)$ algebra: $\hat{\sigma}_{k}^{( \pm)}=\hat{x}_{k} \pm i \hat{y}_{k}$. $\hat{U}_{\mathrm{QMF}}$ transformation of CSA and ladder operators can be thought of as reorientation of individual quantization axes.

\section{Qubit mean-field Hamiltonians of higher classes}

Due to distinguishability of qubits, higher classes of qubit MF-solvable Hamiltonians are somewhat simpler in their operator formulation than those of indistinguishable particles. These higher classes were reviewed extensively in the context of measurement problem in quantum computing. ${ }^{9}$ It is easy to illustrate a higher class MFsolvable Hamiltonian starting with a simple two-qubit example: $\hat{H}_{\mathrm{QMF}, 2}=\hat{x}_{2}+\hat{y}_{2} \hat{z}_{1}$. Here, all eigenstates can be taken as products $\left|\phi_{1} \phi_{2}\right\rangle$, where $\phi_{i}$ 's are eigenstates 
for a single-qubit operator corresponding to the $i^{\text {th }}$ qubit. One can easily confirm that the following 4 products are eigenstates of $\hat{H}_{\mathrm{QMF}, 2}$

$$
\left\{\left|\uparrow_{z} \uparrow_{x+y}\right\rangle,\left|\uparrow_{z} \downarrow_{x+y}\right\rangle, \quad\left|\downarrow_{z} \uparrow_{x-y}\right\rangle,\left|\downarrow_{z} \downarrow_{x-y}\right\rangle\right\},
$$

where $\left|\uparrow_{q}\right\rangle\left(\left|\downarrow_{q}\right\rangle\right)$ corresponds to the eigenstate of $\hat{q}$ with $+1(-1)$ eigenvalue. Note that depending on the eigenstate of the $\hat{z}_{1}$ operator the eigenstate for the second qubit changes.

This consideration can be generalized to the $N$-qubit case, where each qubit can have its quantization axis defined by a single qubit rotation and other qubits can have their own quantization axes that depend on whether previous qubit eigenstates are up or down along their quantization axes.

Due to particle distinguishability, the criterion for the Hamiltonian to be MF-solvable can be formulated using the procedure of consequential integration of qubit variables with checking that there is always at least one single-particle operator commuting with each reduced Hamiltonian. ${ }^{9}$ For $\hat{H}_{\mathrm{QMF}, 2}$, there is $\hat{z}_{1}$ that commutes with $\hat{H}_{\mathrm{QMF}, 2}$, the reduced Hamiltonian is

$$
\hat{H}_{r}=\left\langle\phi_{1}\left|\hat{H}_{\mathrm{QMF}, 2}\right| \phi_{1}\right\rangle=\hat{x}_{2} \pm \hat{y}_{2},
$$

where \pm is determined by the choice of $\phi_{1}$, but in any case, there is a single qubit operator commuting with $\hat{H}_{r}$, which is $\hat{H}_{r}$ because of its single-qubit nature.

\section{CONCLUSIONS}

We have proposed a general Lie-algebraic approach that provided necessary and sufficient conditions for defining the MF-solvable Hamiltonians. The generality of our consideration stems from its applicability to realization of the electronic Hamiltonian in any reductive Lie algebra. There are two main components of the algebraic consideration: 1) the largest commuting set of operators forming the Cartan sub-algebra and 2) the unitary transformations based on the universal covering Lie group obtained by exponentiating the Lie algebra used for the Hamiltonian realization. Compared to previous considerations, our framework goes beyond simple quadratic fermionic Hamiltonians and also includes the Hamiltonians where multiple unitary $\mathrm{MF}$ rotations are needed to obtain all MF eigenstates.

The main practical value of the new definition is that it allows one to find decompositions of any Hamiltonian into MF-solvable components. Such components has already been used in developing efficient quantum computing measurement scheme ${ }^{3}$ and will be generally useful in quantum simulations. Another advantage of the MF decomposition is in selecting the Lie algebra realization for the Hamiltonian of interest so that there is a predominant MF-solvable part. In this case doing perturbative expansions around the predominant MF-solvable part can provide an accurate treatment of many-body effects. Accuracy of this perturbative expansion will depend on magnitudes of residual couplings between neighbouring MF eigenstates compared to energy differences between these states.

\section{ACKNOWLEDGEMENTS}

A.F.I. is grateful to I.G. Ryabinkin, A.V. Zaitsevskii, and V.N. Staroverov for stimulating discussions and acknowledges financial support from the Google Quantum Research Program, Zapata Computing, and the Natural Sciences and Engineering Research Council of Canada.

\section{APPENDIX A: COMPACT LIE ALGEBRAS FROM ANTI-HERMITIAN OPERATORS}

Here we will show that any set of anti-hermitian operators that is closed with respect to the commutation operation, and whose elements have bounded spectrum, represents a compact Lie algebra. Also, such operators can be used to realize hermitian Hamiltonians.

First, any set of anti-hermitian operators closed with respect to commutation form a real Lie algebra. If $\left\{\hat{A}_{i}\right\}$ 's are basis elements of the Lie algebra of anti-hermitian operators, then

$$
\left[\hat{A}_{i}, \hat{A}_{j}\right]=\sum_{k} \xi_{i j}^{(k)} \hat{A}_{k} .
$$

Let us obtain conjugate transposed version of this identity

$$
\begin{aligned}
{\left[\hat{A}_{i}, \hat{A}_{j}\right]^{\dagger} } & =\sum_{k}\left(\xi_{i j}^{(k)}\right)^{*} \hat{A}_{k}^{\dagger} \\
-\left[\hat{A}_{i}, \hat{A}_{j}\right] & =-\sum_{k}\left(\xi_{i j}^{(k)}\right)^{*} \hat{A}_{k} \\
{\left[\hat{A}_{i}, \hat{A}_{j}\right] } & =\sum_{k}\left(\xi_{i j}^{(k)}\right)^{*} \hat{A}_{k} .
\end{aligned}
$$

Comparing Eqs. (58) and (61), it is clear that the structural constants must be real, $\operatorname{Im}\left(\xi_{i j}^{(k)}\right)=0$.

Second, to establish that a real Lie algebra is compact there should be a strictly positive definite bilinear form $(.,$.$) that satisfies the following condition$

$$
([X, Y], Z)+(Y,[X, Z])=0,
$$

where $X, Y, Z$ are elements of the Lie algebra. It was shown that the form $(X, Y)=\operatorname{Re} \operatorname{Tr}\left(X Y^{\dagger}\right)$, satisfies these conditions for a set elements forming a real Lie algebra and closed under conjugate transpose. ${ }^{24}$ The only extra condition we need to introduce is that our operators have bounded spectra to avoid problems with the trace operation.

To use anti-hermitian operators for realizing the hermitian Hamiltonians $\hat{H}$ one can instead realize the antihermitian counterpart of the Hamiltonian $i \hat{H}$ and then 
multiply the obtained realization by $(-i)$. This extra factor will not change the validity of the maximal tori theorem applied to the compact part of the $\hat{H}$ realization.

The Hamiltonians that can be expressed using only elements of the compact Lie algebra $\mathcal{A}$ (no quadratic and higher order terms in Eq. (1)) are MF-solvable. This statement relies on the maximal tori theorem for the compact Lie groups and algebras. ${ }^{25}$ The maximal tori theorem guarantees that for any compact Lie algebra $\mathcal{A}$, any of its elements can be transformed to CSA elements by a unitary transformation taken from the corresponding Lie group (Eq. (5))

$$
\hat{U}\left[\sum_{k=1}^{|\mathcal{A}|} c_{k} \hat{A}_{k}\right] \hat{U}^{\dagger}=\sum_{l=1}^{|\mathcal{C}|} b_{l} \hat{C}_{l},
$$

where $c_{k}$ and $b_{l}$ are all real (or purely imaginary) coefficients, and $|\mathcal{C}|$ is the number of CSA elements.

\section{APPENDIX B: PARTIAL AND FULL MF-SOLVABILITY}

We would like to illustrate that there are partially MFsolvable Hamiltonians. In other words, only a part of their eigenstates can be obtained using MF unitary transformations, and the rest of their spectra require going beyond the MF transformations. Comments on the general procedure establishing full or partial MF-solvability of a Hamiltonian will follow after the example.

An example of a 2-electron Hamiltonian that is partially MF-solvable can be constructed using the condition that its eigenstates are product states if the first orbital is occupied

$$
\hat{H}=\hat{a}_{1}^{\dagger} \hat{a}_{1}+\hat{H}_{\text {non-MF }}\left(1-\hat{a}_{1}^{\dagger} \hat{a}_{1}\right)
$$

where a possible choice of the part that is not solvable by MF rotations is

$$
\begin{aligned}
\hat{H}_{\mathrm{non}-\mathrm{MF}} & =0.43 \hat{a}_{2}^{\dagger} \hat{a}_{2}+0.15 \hat{a}_{2}^{\dagger} \hat{a}_{3}+0.81 \hat{a}_{2}^{\dagger} \hat{a}_{4}+0.64 \hat{a}_{3}^{\dagger} \hat{a}_{2} \\
& -0.15 \hat{a}_{3}^{\dagger} \hat{a}_{2}^{\dagger} \hat{a}_{3} \hat{a}_{2}+0.54 \hat{a}_{3}^{\dagger} \hat{a}_{2}^{\dagger} \hat{a}_{4} \hat{a}_{2}-0.86 \hat{a}_{3}^{\dagger} \hat{a}_{2}^{\dagger} \hat{a}_{4} \hat{a}_{3} \\
& +0.89 \hat{a}_{3}^{\dagger} \hat{a}_{3}+0.21 \hat{a}_{3}^{\dagger} \hat{a}_{4}+0.66 \hat{a}_{4}^{\dagger} \hat{a}_{2}+ \\
& +0.51 \hat{a}_{4}^{\dagger} \hat{a}_{2}^{\dagger} \hat{a}_{3} \hat{a}_{2}+0.76 \hat{a}_{4}^{\dagger} \hat{a}_{2}^{\dagger} \hat{a}_{4} \hat{a}_{2}+0.05 \hat{a}_{4}^{\dagger} \hat{a}_{2}^{\dagger} \hat{a}_{4} \hat{a}_{3} \\
& +0.45 \hat{a}_{4}^{\dagger} \hat{a}_{3}+1.18 \hat{a}_{4}^{\dagger} \hat{a}_{3}^{\dagger} \hat{a}_{3} \hat{a}_{2}+0.25 \hat{a}_{4}^{\dagger} \hat{a}_{3}^{\dagger} \hat{a}_{4} \hat{a}_{2} \\
& -0.16 \hat{a}_{4}^{\dagger} \hat{a}_{3}^{\dagger} \hat{a}_{4} \hat{a}_{3}+0.68 \hat{a}_{4}^{\dagger} \hat{a}_{4} .
\end{aligned}
$$

A general procedure to establish Hamiltonian's MFsolvability would require a search for a MF unitary that provides at least one eigenstate of the given Hamiltonian. Minimizing variance of the Hamiltonian on state $|0\rangle$ containing some number of particles (i.e. physical vacuum) with respect to MF unitaries $\hat{U}$

$$
\hat{U}_{1}=\arg \min _{\hat{U}}\left\langle 0\left|\hat{U}^{\dagger} \hat{H}^{2} \hat{U}\right| 0\right\rangle-\left\langle 0\left|\hat{U}^{\dagger} \hat{H} \hat{U}\right| 0\right\rangle^{2}
$$

allows one to determine whether at least one MF eigenstate can be generated by $\hat{U}_{1}$, which is the case if the variance can be lowered by $\hat{U}_{1}$ to zero. If the variance cannot be lowered to zero, then the Hamiltonian is not MF solvable. Since the optimization is not linear, there can be complications related to finding a local minimum that does not correspond to zero variance even if zero variance exists as a global minimum within the space of all MF-unitaries. A random sampling of initial amplitudes of the MF rotations can be used to address this difficulty.

If a zero-variance MF transformation is found, the next step is to determine whether this transformation provides all or only a subset of all eigenstates. Applying the MF transformation to the Hamiltonian $\tilde{H}=\hat{U}_{1}^{\dagger} \hat{H} \hat{U}_{1}$ can answer this question: if $\tilde{H}=\tilde{F}_{1}\left(\hat{C}_{k}\right)$ contains only CSA elements then $\hat{H}$ is MF-solvable class 1 , whereas if $\tilde{H}$ contains also a non-CSA part then further analysis is needed because $\hat{U}_{1}$ does not provide all eigenstates of $\hat{H}$.

To go beyond $\hat{U}_{1}$ MF-eigenstates, let us assume that $\tilde{H}=\tilde{F}_{1}\left(\hat{C}_{k}\right)+\tilde{F}_{2}\left(\hat{A}_{k^{\prime}}\right)$, where $\tilde{F}_{2}$ is some polynomial over general Lie algebra elements (i.e. the non-CSA part). To determine whether there are other MF unitaries that can provide additional eigenstates one needs to factorize $\tilde{F}_{1}\left(\hat{C}_{k}\right)=F_{1}\left(\hat{C}_{k}\right) \hat{P}_{1}\left(\hat{C}_{k}\right)$ to projector $\hat{P}_{1}\left(\hat{C}_{k}\right)$ that defines the eigensubspace and an additional function $F_{1}\left(\hat{C}_{k}\right)$ (similar to Eq. (15)). The complimentary projector $\hat{P}_{1}^{\perp}=1-\hat{P}_{1}$ can be identified and factorized from $\tilde{F}_{2}\left(\hat{A}_{k^{\prime}}\right)=H_{2}\left(\hat{A}_{k^{\prime}}\right) \hat{P}_{1}^{\perp}$. These factorization are always possible since $\tilde{H}$ has MF-eigenstates decoupled from the rest of the spectrum. To identify new MF rotations one needs to repeat the consideration that led to $\hat{U}_{1}$ starting from searching the minimum of the variance for the $H_{2}\left(\hat{A}_{k^{\prime}}\right)$. This process as before can lead to three outcomes: 1) no MF rotations with zero variance are found, 2) zero-variance MF $\hat{U}_{2}$ is found, and it transforms $\hat{H}_{2}$ to a purely CSA polynomial, and 3) zero-variance MF $\hat{U}_{2}$ is found but it transforms $\hat{H}_{2}$ to a linear combination of a purely CSA and non-CSA polynomials. The first outcome means the end of the algorithm concluding only partial MF-solvability of $\hat{H}$. The algorithm can end in the second case as well, but with the conclusion that $\hat{H}$ is class-2 MF-solvable and its unitary rotations are $\hat{U}_{1}$ and $\hat{U}_{1} \hat{U}_{2}$. Finally, in the third case the algorithm continues to search for other MF unitaries in the non-CSA part of the transformed $\hat{H}_{2}$. There are two possible outcomes of this search: either $\hat{H}$ is partially MF-solvable or it is class $>2$ MF-solvable.

\section{APPENDIX C: NON-TRIVIAL FERMIONIC MF-SOLVABLE HAMILTONIANS}

Here, we present examples of class-1 and -2 MFsolvable Hamiltonians, for simplicity, they are limited to three spin-less orbitals. In both examples, CSA elements are taken as orbital occupation operators $\hat{E}_{p}^{p}$. 
Class 1: Using form of Eq. (12) we set to zero all linear terms $c_{k}=0$, to have real coefficients in the Hamiltonian, $\phi_{p q}=0$ in the MF rotation of Eq. (38), while $\theta_{p q}$ and $d_{k k^{\prime}}$ are chosen as follow

$$
\begin{aligned}
& \left(d_{12}, d_{13}, d_{23}\right)=(-27,-9,-9) \\
& \left(\theta_{12}, \theta_{13}, \theta_{23}\right) \approx(-2.214,-1.459,-2.214)
\end{aligned}
$$

This setup produces the following two-electron MFsolvable Hamiltonian

$$
\begin{aligned}
\hat{H}_{\mathrm{MF}, 1}= & 11 \hat{a}_{2}^{\dagger} \hat{a}_{1}^{\dagger} \hat{a}_{2} \hat{a}_{1}+4 \hat{a}_{2}^{\dagger} \hat{a}_{1}^{\dagger} \hat{a}_{3} \hat{a}_{1}+4 \hat{a}_{2}^{\dagger} \hat{a}_{1}^{\dagger} \hat{a}_{3} \hat{a}_{2} \\
& +4 \hat{a}_{3}^{\dagger} \hat{a}_{1}^{\dagger} \hat{a}_{2} \hat{a}_{1}+17 \hat{a}_{3}^{\dagger} \hat{a}_{1}^{\dagger} \hat{a}_{3} \hat{a}_{1}+8 \hat{a}_{3}^{\dagger} \hat{a}_{1}^{\dagger} \hat{a}_{3} \hat{a}_{2} \\
& +4 \hat{a}_{3}^{\dagger} \hat{a}_{2}^{\dagger} \hat{a}_{2} \hat{a}_{1}+8 \hat{a}_{3}^{\dagger} \hat{a}_{2}^{\dagger} \hat{a}_{3} \hat{a}_{1}+17 \hat{a}_{3}^{\dagger} \hat{a}_{2}^{\dagger} \hat{a}_{3} \hat{a}_{2} .
\end{aligned}
$$

Class 2: For simplicity, we keep $\hat{U}_{1}=I$ and $\hat{P}_{1}=\hat{E}_{1}^{1}$ in Eq. $\hat{U}_{2}$ 's only non-zero rotation is $\theta_{23}=1.55$ so that $\hat{U}_{2}$ commutes with $\hat{P}_{1} . F_{1}$ and $F_{2}$ are linear functions of CSA elements with coefficients

$$
\begin{aligned}
& F_{1}:\left\{c_{k}\right\}=\{0.44,0.61,0.95\} \\
& F_{2}:\left\{c_{k}\right\}=\{0.34,0.69,0.23\} .
\end{aligned}
$$

These parameters generate the two-electron class-2 MFsolvable Hamiltonian whose all eigen-states are Slater determinants

$$
\begin{aligned}
\hat{H}_{\mathrm{MF}, 2} & =-0.61 \hat{a}_{2}^{\dagger} \hat{a}_{1}^{\dagger} \hat{a}_{2} \hat{a}_{1}-0.95 \hat{a}_{3}^{\dagger} \hat{a}_{1}^{\dagger} \hat{a}_{3} \hat{a}_{1}+0.23 \hat{a}_{2}^{\dagger} \hat{a}_{1}^{\dagger} \hat{a}_{2} \hat{a}_{1} \\
& +0.01 \hat{a}_{2}^{\dagger} \hat{a}_{1}^{\dagger} \hat{a}_{3} \hat{a}_{1}+0.23 \hat{a}_{2}^{\dagger} \hat{a}_{2}+0.01 \hat{a}_{2}^{\dagger} \hat{a}_{3}+0.01 \hat{a}_{3}^{\dagger} \hat{a}_{1}^{\dagger} \hat{a}_{2} \hat{a}_{1} \\
& +0.69 \hat{a}_{3}^{\dagger} \hat{a}_{1}^{\dagger} \hat{a}_{3} \hat{a}_{1}+0.01 \hat{a}_{3}^{\dagger} \hat{a}_{2}+0.69 \hat{a}_{3}^{\dagger} \hat{a}_{3}
\end{aligned}
$$

where the first two terms are $F_{1}\left(\hat{C}_{k}\right) \hat{P}_{1}$.
* artur.izmaylov@utoronto.ca

1 T. Helgaker, P. Jørgensen, and J. Olsen, Molecular Electronic-Structure Theory (John Wiley \& Sons, Ltd, Chichester, UK, 2000).

2 H. Barnum, E. Knill, G. Ortiz, and L. Viola, Phys. Rev. A 68, 195 (2003).

3 T.-C. Yen and A. F. Izmaylov, arXiv.org (2020), $2007.01234 \mathrm{v} 1$.

4 R. Somma, H. Barnum, G. Ortiz, and E. Knill, Phys. Rev. Lett. 97, 467 (2006).

5 R. D. Somma, J. Math. Phys. 60, 112202 (2019).

6 S. B. Bravyi and A. Y. Kitaev, Ann. Phys. 298, 210 (2002)

7 J. T. Seeley, M. J. Richard, and P. J. Love, J. Chem. Phys. 137, 224109 (2012).

8 Here, by a product we refer to a proper tensor product between states from different single-particle spaces. For example, indistinguishable boson/fermion particles requires symmetrized/anti-symmetrized products.

9 A. F. Izmaylov, T. C. Yen, and I. G. Ryabinkin, Chem. Sci. 10, 3746 (2019).

10 B. Poirier, Phys. Rev. A 56, 120 (1997).

11 I. G. Ryabinkin, S. N. Genin, and A. F. Izmaylov, J. Chem. Phys. 149, 214105 (2018).

12 P. Ring and P. Schuck, The Nuclear Many-Body Problem (Springer-Verlag, New York, NY, 1980).

13 E. Lieb, T. Schultz, and D. Mattis, Ann. of Phys. 16, 407
(1961).

14 P. Jordan and E. Wigner, Z. Phys. 47, 631 (1928).

15 A. Tranter, S. Sofia, J. Seeley, M. Kaicher, J. McClean, R. Babbush, P. V. Coveney, F. Mintert, F. Wilhelm, and P. J. Love, Int. J. Quantum Chem. 115, 1431 (2015).

16 R. Gilmore, Lie Groups, Physics, and Geometry: An Introduction for Physicists, Engineers and Chemists (Cambridge University Press, 2008).

17 A. O. Barut and R. Raczka, Theory of Group Representations and Applications (Polish Scientific Publisher, Warszawa, Poland, 1980).

18 A. F. Izmaylov, M. Díaz-Tinoco, and R. A. Lang, Physical Chemistry Chemical Physics 22, 12980 (2020).

19 H. Fukutome, Prog. Theor. Phys. 65, 809 (1981).

20 J. Paldus and C. R. Sarma, J. Chem. Phys. 83, 5135 (1985).

21 A. Chapman and S. Flammia, Quantum 4, $278(2020)$.

${ }^{22}$ K. Setia and J. D. Whitfield, J. Chem. Phys. 148, 164104 (2018).

23 V. Havlíček, M. Troyer, and J. D. Whitfield, Phys. Rev. A 95, 032332 (2017).

24 A. W. Knapp, in Lie groups beyond an introduction (Second Edition, Progress in Mathematics, Vol. 140 Birkhäuser, Boston, 2002) pp. 57-58.

25 B. C. Hall, in Lie Groups, Lie Algebras, and Representations: An Elementary Introduction (Springer, Switzerland, 2015) p. 314. 